\title{
Comunicação Interatrial do Tipo Seio Coronário, Comunicação Interventricular e Ausência de Veia Cava Superior Esquerda
}

\author{
Fábio Alves Almeida, Carlos Augusto Cardoso Pedra, Carlos Augusto de Jesus, \\ Simone Rolim Femandes Fontes Pedra, Valmir Femandes Fontes, Luís Carlos Bento de Sousa
}

São Paulo, SP

\begin{abstract}
Relatamos um caso raro de um paciente de 21 meses, portador de comunicação interatrial do tipo seio coronário, associada a comunicação interventricular perimembranosa, e ausência de veia cava superior esquerda. O diagnóstico foi realizado através da ecocardiografia e confirmado pela angiografia. O paciente foi operado sem intercorrências, ambos os defeitos foram fechados com patch de pericárdio bovino e o fluxo das veias coronárias ficou direcionado para o átrio esquerdo. Um ecocardiograma mostrou ausência de shunt residual através dos defeitos.
\end{abstract}

\section{Coronary Sinus Atrial Septal Defect and Ventricular Septal Defect with no Left Superior Vena Cava}

We report a rare case of a 21 month old child with a coronary sinus atrial septal defect associated with perimembranous ventricular septal defect and no left superior vena cava. The diagnosis was made by transthoracic echocardiogram and confirmed by angiography. The patient was operated on uneventfully, both defects were closed with bovine pericardial patches and the flow from the coronary veins was directed towards the left atrium. An echocardiogram revealed complete closure of both defects.
A comunicação interatrial (CIA) corresponde a cerca de $10-15 \%$ de todas as cardiopatias congênitas ${ }^{1}$. Os defeitos localizados na lâmina da fossa oval são responsáveis pela absoluta maioria da CIAs. Menos freqüente são as CIAs do tipo ostium primum (que na verdade devem ser encaradas como defeitos do septo atrioventricular com junção atrioventricular comum) e do tipo seio venoso ${ }^{1}$. A CIA do tipo seio coronário (SC), também chamada de SC parcialmente ou totalmente sem teto, é uma doença rara ${ }^{2-8}$, resultante de uma incompleta formação da junção átrio venosa $^{2}$. Geralmente está associada a cardiopatias congênitas complexas e persistência da veia cava superior esquerda (VCSE) $)^{2-7}$, sendo excepcional seu encontro com má formação isolada ${ }^{4}$. Neste artigo, descrevemos um caso de CIA do tipo SC associada a comunicação interventricular (CIV) perimembranosa e ausência de VCSE, sendo diagnosticada através da ecocardiografia e confirmada pela angiografia.

Instituto Dante Pazzanese de Cardiologia - São Paulo

Correspondência: Fábio Alves Almeida - Instituto Dante Pazzanese de Cardiologia - Av. Dr. Dante Pazzanese, 500 - 04012-180 - São Paulo, SP

Recebido para publicação em 24/6/98

Aceito em 28/7/98

\section{Relato do caso}

Criança de 21 meses, sexo feminino, peso de $8 \mathrm{~kg}$, foi encaminhada ao nosso serviço para avaliação de doença cardíaca congênita. Apresentava história de dispnéia aos esforços, dificuldade para ganhar peso, broncopneumonias de repetição e insuficiência cardíaca de difícil controle clínico. O exame físico revelou leve taquipnéia e ausência de cianose. Foram auscultados um sopro sistólico ejetivo (++/6+) em foco pulmonar e um sopro sistólico regurgitativo $(+++/ 6+)$ ao longo do bordo esternal esquerdo baixo. A $2^{\text {a }}$ bulha apresentava desdobramento fixo curto com componente pulmonar de intensidade aumentada. O fígado era palpável a $3 \mathrm{~cm}$ do rebordo costal direito. O eletrocardiograma revelou ritmo sinusal, com SAQRS a $\pm 180^{\circ}$ e sobrecarga biventricular com predomínio de ventrículo direito. A radiografia de tórax mostrou situs solitus, ponta para a esquerda, tronco da pulmonar abaulada, área cardíaca e fluxo pulmonar aumentados. Um ecocardiograma realizado fora de nossa Instituição revelou CIA tipo ostium secundum e CIV amplas com shunt esquerdo-direito. Um ecocardiograma bidimensional transtorácico com mapeamento de fluxo a cores realizado em nossa Instituição mostrou situs solitus 


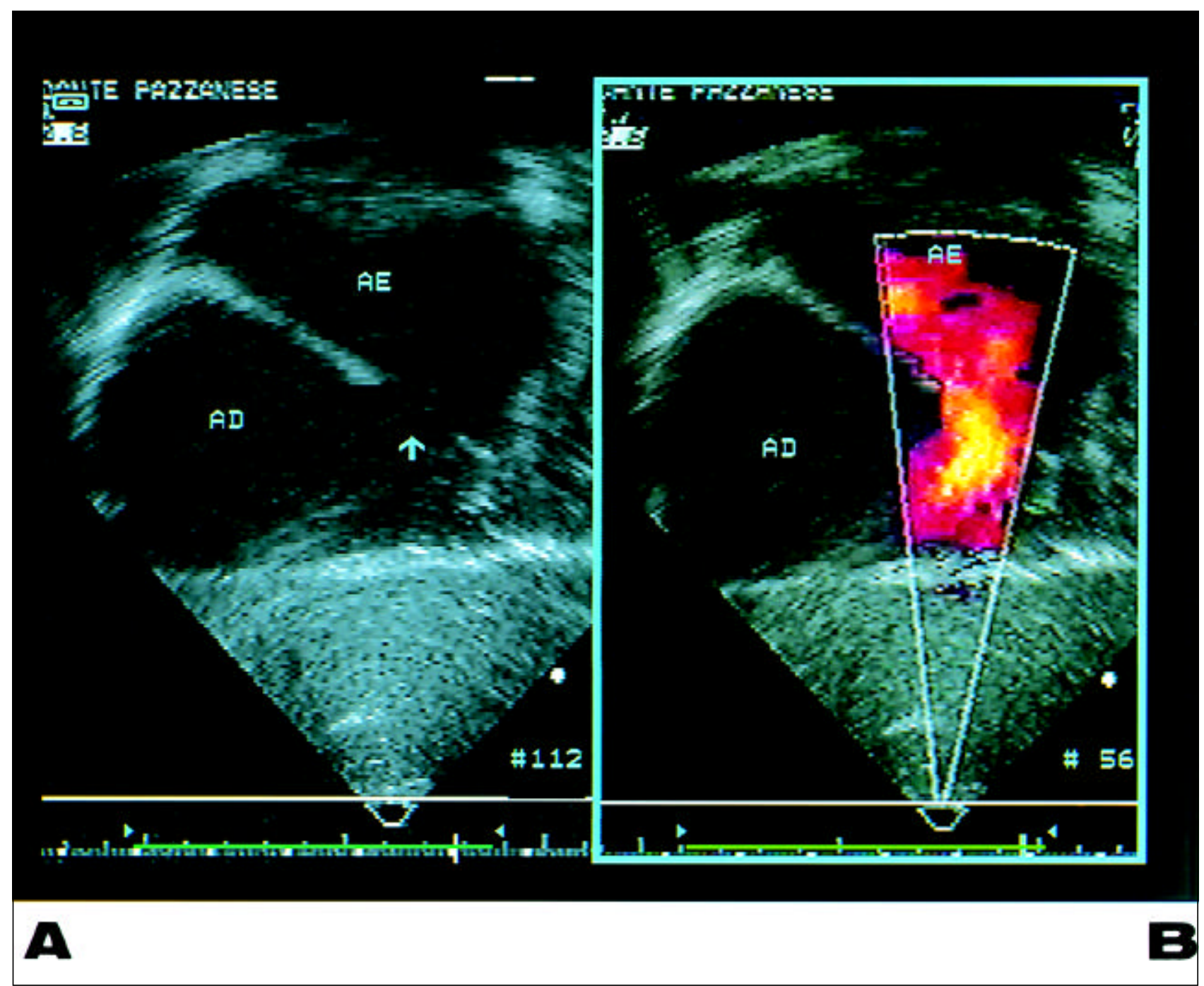

Fig. 1 - Ecocardiograma bidimensional com Doppler colorido. A) projeção subcostal mostrando ausência do teto do seio coronário permitindo uma ampla comunicação entre os átrios em porções baixas do septo interatrial; B) projeção subcostal com mapeamento de fluxo a cores mostrando o shunt esquerdo direito através do defeito. AE- átrio esquerdo; $\mathrm{AD}$ átrio direito; seta- comunicação interatrial tipo seio coronário.

com levocardia; defeito do septo atrial tipo SC amplo com shunt do átrio esquerdo (AE) para oátrio direito (AD) (fig. 1); defeito do septo interventricular perimembranoso, parcialmente ocluído pelo folheto septal da valva tricúspide, com shunt de ventrículo esquerdo(VE) para o direito(VD); ausência de VCSE; aumento importante das cavidades direitas com câmaras esquerdas de dimensões normais; dilatação importante do tronco da artéria pulmonar (TP) e ramos e sinais de hipertensão arterial pulmonar.

O cateterismo cardíaco foi indicado para avaliação do grau de hipertensão pulmonar e para confirmação diagnóstica, já que se tratava de um caso não usual. As medidas pressóricas (em mmHg) foram: ADm: 2; VD: 51/2; TP: 51/15 (m: 32); Ao: 60/40 (m:49); VE: 60/6; AEm: 3 . O estudo oximétrico demonstrou: $\mathrm{VCS}=66 \% ; \mathrm{AD}=88 \% ; \mathrm{VD}=88 \%$; $\mathrm{TP}=91 \%$; e Ao $=96 \%$. Realizados cálculos de fluxo e resistência sistêmica e pulmonar que demonstraram: QP/QS de 5:1 e RVP/RVS de 0,12. Oestudo angiográfico confirmou os dados ecocardiográficos (fig. 2).
O paciente foi encaminhado para tratamento cirúrgico e os achados intraoperatórios corroboraram com os diagnósticos ecocardiográficos e angiográfico. Ambos os defeitos foram fechados com patches de pericárdio bovino e a drenagem do fluxo proveniente das veias coronárias foi deixada em direção ao AE. O pós operatório transcorreu sem intercorrências e o paciente teve alta hospitalar uma semana após a cirurgia. O ecocardiograma de alta revelou oclusão total dos defeitos.

\section{Discussão}

A singularidade deste caso reside basicamente em três fatores: no diagnóstico da CIA tipo SC que por si só é uma cardiopatia congênita rara ${ }^{1-8}$; na sua associação com outra cardiopatia não complexa como a CIV e na ausência da VCSE, o que também não é comum. Do ponto de vista embriológico, a CIA do tipo SC provavelmente decorre de uma septação incompleta da junção átrio venosa entre a 

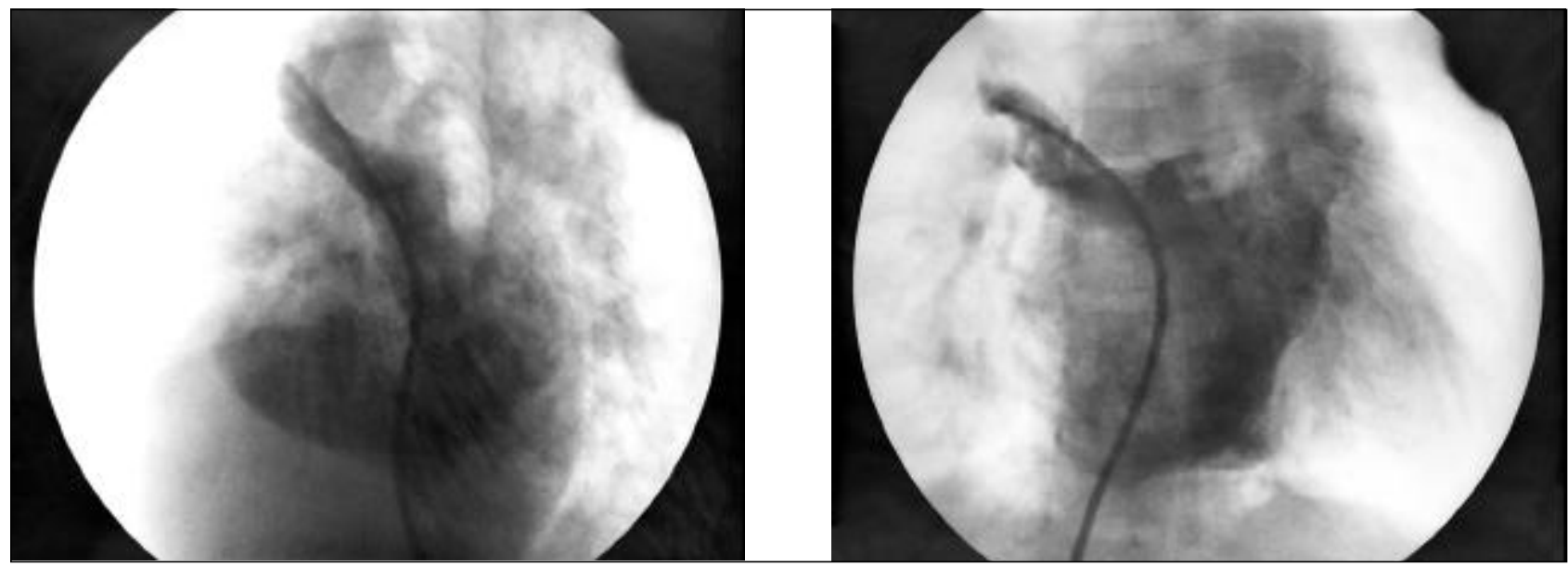

Fig. 2 - Angiografia em veia pulmonar superior direita, mostrando passagem de contraste do átrio esquerdo para o átrio direito através de amplo defeito do septo interatrial localizado em porções inferiores do septo. Notem que o cateter cruza o septo interatrial através de forame oval pérvio e não através da comunicação interatrial. A) projeção hepatoclavicular (4 câmaras); B) projeção póstero-anterior (de frente).

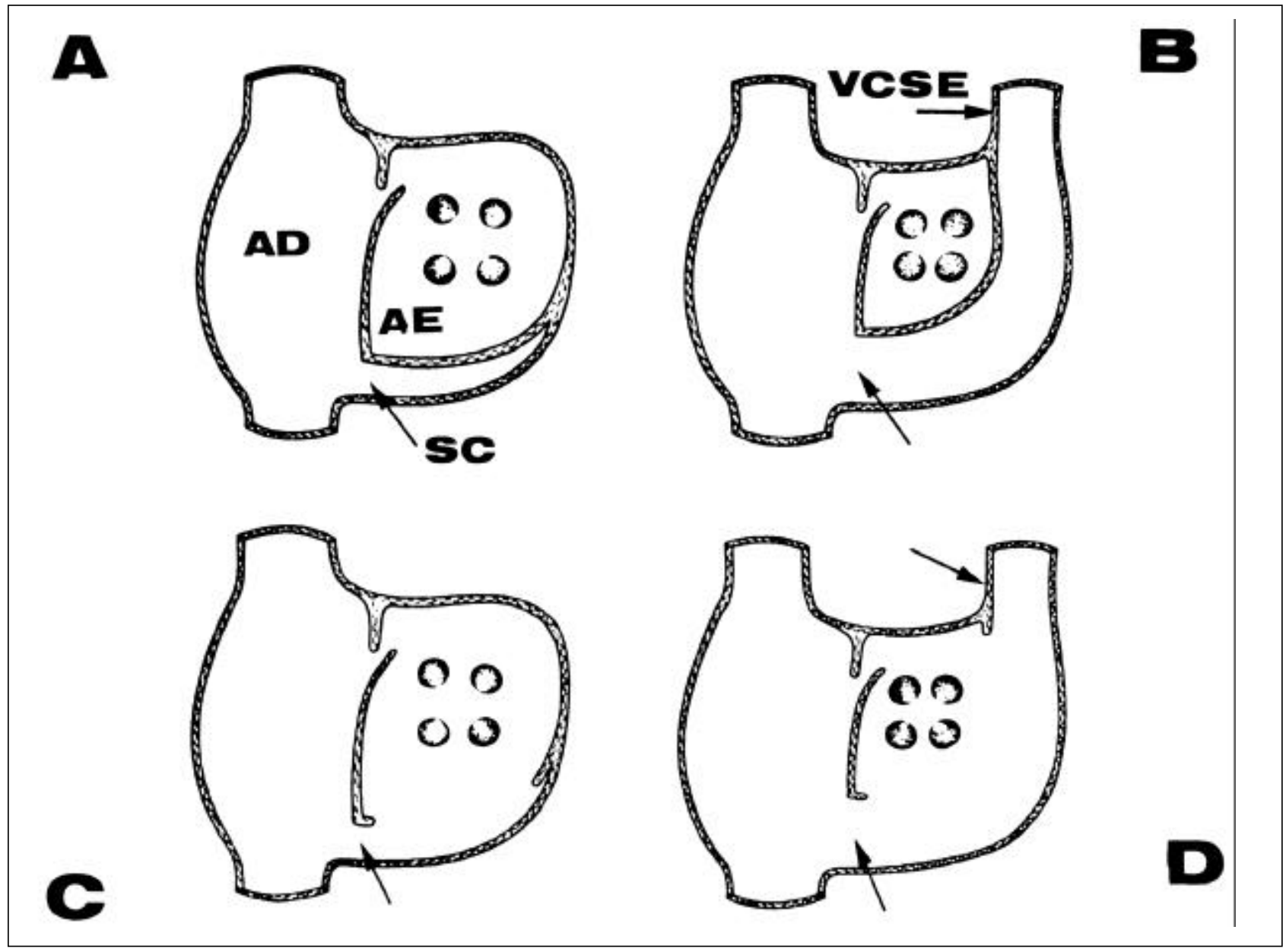

Fig. 3 - Diagrama da ausência parcial ou total do seio coronário ${ }^{7}$. A) situação anatômica normal; B) veia cava superior esquerda persistente conectada em seio coronário; C) ausência parcial do teto do seio coronário sem a coexistência de veia cava superior esquerda persistente; D) ausência total do teto do seio coronário associada a veia cava superior esquerda persistente (complexo de Raghib). AD- átrio direito; AE- átrio esquerdo; SC- seio coronário; VCSE- veia cava superior esquerda persistente.

veia cardinal esquerda e $\mathrm{AE} \mathrm{AE}^{2}$. Isto explicaria por que a persistência da VCSE persistente está invariavelmente associada a tal tipo de CIA. Utilizando este conceito, Raghib e $\mathrm{col}^{3}$ descreveram um caso em 1965 em que havia persistência da VCSE terminando no AE e ausência total do SC. Essa descrição corresponde à síndrome clássica. Por vezes, os termos 
SC completamente sem teto e SC ausente são usados como sinônimos. Essa nomenclatura é debatida por alguns autores já que se o orifício do SC é identificável, e se este é considerado parte do seio, não é completamente justificável nomear esta situação como ausência completa do $\mathrm{SC}^{2}$. Por outro lado, quando não é identificado um orifício do SC ou uma parede separando o SC do AE e na presença de uma VCSE, o termo ausente pode ser usado ${ }^{2}$. A fim de se evitar estes desentendidos, é preferível referir-se a lesão com um termo meramente anatômico, como ausência parcial ou total do teto do $\mathrm{SC}^{2}$. A ausência parcial do SC é considerada por alguns autores como uma forma frustada do complexo de Raghib ${ }^{4,7}$. Apesar da presença da VCSE ser quase uma constante nestas situações, alguns casos têm sido descritos na sua ausência ${ }^{4}$. A ausência parcial ou total do SC está representada em forma de diagrama na figura 3.

A CIA tipo SC está comumente associada a cardiopatias complexas, como isomerismos, conexões atriais discordantes, dupla via de saída de VD, atresia tricúspide entre outras $^{2,5,6}$. Cardiopatias menos complexas como tetralogia de Fallot, defeitos do septo atrioventricular e coartação de aorta também podem coexistir ${ }^{6,8}$. Poucos casos têm sido descritos com CIV associada, sendo excepcional seu achado como doença isolada ${ }^{4}$. Às vezes uma CIA tipo SC pode se confluir com uma CIA tipo ostium secundum ocasionando um amplo defeito no septo interatrial ${ }^{6}$.

A repercussão clínica da CIA tipo $\mathrm{SC}$ depende de suas dimensões e de outras lesões associadas que promoveriam shunts esquerdo/direito (E/D) ou em sentido inverso ao nível atrial. A ausência completa do teto do SC é um substrato anatômico que propicia a ocorrência de grandes shunts. Por outro lado, a presença de pequenas fenestrações neste local pode restringir o fluxo entre os átrios.

Do ponto de vista ecocardiográfico, o defeito não é fácil de ser delineado devido a natureza curvilínea posterior extensa do $\mathrm{SC}^{5}$. O melhor plano para definição do diagnóstico é o subcostal, onde podemos visibilizar adequadamente a região posterior de ambos os átrios. Na CIA tipo SC, o teto do SC encontra-se ausente (de forma parcial ou total), promovendo uma comunicação entre o que seria o assoalho do $\mathrm{AE}$ e o $\mathrm{SC}^{5}$. O shunt $\mathrm{E} / \mathrm{D}$ (ou em sentido inverso) através dos átrios é documentado através do mapeamento de fluxo a cores. A lesão também pode ser visibilizada na projeção para esternal alta, eixo maior (longitudinal), onde podemos documentar a dilatação do seio. O estudo ecocardiográfico desta área posterior do coração nos planos referidos pode levar a fenômeno de drop out, o que acarretaria em falsa impressão de ausência do teto do $\mathrm{SC}^{5}$. O diagnóstico de VCSE deve ser sempre pesquisado através do modo bidimensional e do mapeamento de fluxo a cores e na sua suspeita, deve ser realizada a ecocardiografia com contraste. Uma solução salina é agitada e injetada em veia calibrosa em membro superior esquerdo, permitindo definir o local de entrada da veia cava esquerda no $\mathrm{AE}^{5}$. Esta técnica tem o potencial de substituir a angiografia para realização do diagnóstico da doença ${ }^{5}$. No caso estudado, a veia inominada era calibrosa e não havia VCSE. O estudo detalhado do SC e de possíveis anomalias sistêmicas assume importância ainda maior em crianças com cardiopatias complexas que são referidas para reparo cirúrgico paliativo ou definitivo, através de anastomoses do tipo cavopulmonar ${ }^{5,7}$. Se a CIA do tipo SC ou a anomalia venosa não foram suspeitadas e reparadas, shunts residuais e/ou cianose podem resultar ou persistir após a cirurgia $^{5,7}$.

O cateterismo cardíaco e a angiografia devem ser realizados dependendo do tipo de lesões associadas, quando há dúvida diagnóstica e necessidade de realização de cálculos de fluxo e resistência. Quando o shunt ocorre da esquerda para a direita, o salto oximétrico é observado entre a VCS e AD, com elevação significativa dos valores oximétricos das amostras colhidas do SC. No caso relatado, o shunt E/D global era de grande magnitude, localizado principalmente ao nível atrial, denotando as grandes dimensões do defeito. A angiografia é realizada de preferência na veia pulmonar superior direita nas projeções hepatoclavicular (ou 4 câmaras) e em póstero-anterior (de frente $)^{4,7}$. Injeções no AE ou no TP com estudo do levograma nas mesmas projeções, também, podem ser empregadas alternativamente ${ }^{4,7}$. Notase passagem do contraste do AE para o AD em porções baixas do septo interatrial. A associação de defeitos do septo atrioventricular pode dificultar o diagnóstico, já que o shunt atrial também ocorre em regiões adjacentes. É aconselhável realizar uma injeção na veia inominada (se estiver presente), ou em jugular interna esquerda ou em veia calibrosa de membro esquerdo a fim de avaliar a presença de VCSE e o local de sua drenagem. Estudo que pode, também, ser realizado nas projeções de frente e de perfil esquerdo ${ }^{7}$.

A abordagem cirúrgica da CIA tipo SC é realizada de diversas formas, dependendo das doenças associadas. Quando há VCSE persistente drenando em AE, o cirurgião pode reconstruir todo o teto do $\mathrm{SC}$ com um patch de pericárdio bovino, redirecionando, assim, o fluxo da VCSE para o $\mathrm{AD}^{6}$. Em casos onde não há VCSE associada, como o aqui relatado, o cirurgião tem a opção de apenas fechar a CIA com um patch, deixando a drenagem do fluxo proveniente do SC e das veias coronárias para o $\mathrm{AE}$, com um grau desprezível de dessaturação sistêmica ${ }^{6}$. O simples fechamento da CIA na presença de veia cava persistente com drenagem em AE pode levar inadvertidamente a shunts E/D significativos, com dessaturação sistêmica, fenômenos tromboembólicos, ocorrência de acidente vascular e abcessos cerebrais ${ }^{7}$. As lesões associadas devem ser corrigidas de forma individualizada.

Em resumo, este relato descreve um caso de CIA tipo $\mathrm{SC}$, associado a CIV e ausência de veia cava esquerda persistente, diagnosticado corretamente através da ecocardiografia e angiografia, com evolução pós-operatória satisfatória. O cardiologista pediátrico deve sempre pesquisar o diagnóstico de CIA tipo SC na vigência de um defeito do septo interatrial amplo associado à dilatação do SC, com ou sem persistência da VCSE. 


\section{Referências}

1. Medeiros Sobrinho JH, Fontes VF, Pontes Jr SC - Defeitos isolados do septo atrial. In: Medeiros Sobrinho JH, Fontes VF, Pontes Jr SC, eds. Cardiopatias congênitas. São Paulo: Sarvier 190: pag: 295-314.

2. Adatia I, Gittenberger-de Groot AC - Unroofed coronary sinus and coronary sinus orifice atresia. Implications for management of complex congenital heart disease. J Am Coll Cardiol 1995; 25: 948-53.

3. Raghib G, Ruttenber HD, Anderson RC et al - Termination of left superior vena cava in left atrium, atrial septal defect, and absense of coronary sinus, a developmental complex. Circulation 1965; 31: 906-18.

4. Freedom RM, Culham JAG, Rowe RD - Left atrial to coronary sinus fenestration (partially unruufed coronary sinus) morphological and angiocardiographic observations. Br Heart J 1981; 46: 63-8.
5. Schmidt KG, Silverman NH - Cross-sectional and contrast echocardiography in the diagnosis of interatrial communications through the coronary sinus. Int $\mathrm{J}$ Cardiol 1987; 16: 193-9.

6. Quagebeur J, Kirklin JW, Pacifico AD, Bargeron LM - Surgical experience with unroofed coronary sinus. Ann Thorac Surg 1979; 27: 418-25.

7. Freedom RM, Mawson JB, Yoo SJ, Benson LN - Anomalies of systemic venous connections, coronary sinus and divided right atrium (cor triatriatum dexter), In: Freedom RM, Mawson JB, Yoo SJ, Benson LN, editors. Congenital heart disease. Textbook of angiography. Armonk, NY. Futura Publishing Co. 1997: 291-337.

8. Allmendinger P, Dear WE, Cooley DA - Atrial septal defect with communication through the coronary sinus. Ann Thorac Surg 1974; 17: 193-6. 